\title{
Vacunas contra covid-19 aplicadas por grupo de edad en Colombia
}

\section{COVID-19 vaccines administered by age group in Colombia}

a Ingeniero. Magister en Gestión de la Tecnología Educativa, Especialista en Administración de la Informática Educativa.

Docente de matemáticas e Investigador, Secretaría de Educación de Soacha, Cundinamarca.

\section{R E S U M E N}

Introducción: e están invirtiendo miles de millones de dólares en la investigación de la patogénesis, el tratamiento y la prevención del COVID-19. Para la prevención, se han desarrollado al menos 90 vacunas con la esperanza de proporcionar inmunidad. Objetivo: exponer el avance de los esquemas completos aplicados contra SARS-COV-2 por grupo de edad en las entidades territoriales de Colombia, en el periodo comprendido entre el 17 de febrero al 21 de agosto 2021. Metodología: estudio de tipo transversal analítico diseñado de manera específica para recoger información sobre vacunas administradas en Colombia por grupo de edad; como fuente de información se tomó el plan nacional de vacunación contra el COVID-19 de la página web del Ministerio de Salud y Protección Social. Resultados: se concluyó que el mayor número de esquemas completos por grupo de edad fueron: 50 a 59 años (2'089.980), 60 a 69 años (1'937.880), y 40 a 49 años (1'480.711). El menor número de esquemas completos por grupo de edad fueron:12 a 19 años (55.113), 20 a 29 años (476.866) y 30 a 39 años (624.909). Conclusión: en la actualidad la vacunación es la maniobra más importante para poner fin a la pandemia. Sin embargo, el arribo de múltiples variantes del SARS-COV-2 con susceptibilidad reducida a la inmunidad inducida por la vacuna y la enfermedad amenaza el progreso.

Palabras clave: COVID-19, SARS-COV-2, pandemia, vacunas.

(C) 2021 Fundación Universitaria de Ciencias de la Salud - FUCS. Este es un artículo Open Access bajo la licencia CC BY-NC-ND (http://creativecommons.org/licenses/by-nc-nd/4.0/).

\section{INFORMACIÓN DEL ARTÍCULO}

Historia del artículo:

Fecha recibido: agosto 30 de 2021 Fecha aceptado: septiembre 22 de 2021

\footnotetext{
Autor para correspondencia.

Jorge Enrique Díaz Pinzón jediazp@unal.edu.co
}

DOI

10.31260/RepertMedCir.01217372.1276 


\section{A BSTRACT}

Introduction: thousands of dollars are being invested for research on COVID 19 pathogenesis, treatment and prevention. To prevent COVID 19, at least 90 vaccines have been developed with the hope for providing immunity. Objective: to describe the progression of SARS-COV-2 full vaccination by age group by territorial entity in Colombia, in the period between February 17 and August 21 2021. Methodology: an analytical cross-sectional study specifically designed to gather information on vaccines administered in Colombia by age group; using the information released in the Ministry of Health and Social Protection webpage based on the COVID 19 national vaccination plan. Results: the highest full vaccination by age group was found in people in the 50 to 59 years (2'089.980), 60 to 69 years (1'937.880), and 40 to 49 years (1'480.711) age groups. The lowest number of completed vaccination protocols by age group was found in the: 12 to 19 years (55.113), 20 to 29 years (476.866) and 30 to 39 years (624.909) age groups. Conclusion: currently vaccination is the most important intervention to bring the pandemic to an end. However, the emergence of multiple SARS-COV-2 variants with reduced susceptibility to vaccineinduced immunity makes the disease to remain a threat to progress.

Key words: COVID-19, SARS-COV-2, pandemic, vaccines.

(C) 2021 Fundación Universitaria de Ciencias de la Salud - FUCS. This is an open access article under the CC BY-NC-ND license (http://creativecommons.org/licenses/by-nc-nd/4.0/).

IN TRODUCCIÓN

Se están invirtiendo varios millones de dólares en la búsqueda de la patogénesis, el tratamiento, y la prevención del COVID-19. Para la prevención se han propuesto cerca de 90 vacunas con la esperanza de proporcionar inmunidad. ${ }^{1}$ En noviembre 2020, tres compañías farmacéuticas informaron que sus vacunas se encontraban en la fase 3 de los ensayos clínicos y publicaron tasas de eficacia preliminares para obtener la aprobación de emergencia de la Administración de Alimentos y Medicamentos (FDA). Se demostró que tenían una eficacia superior a 95\%, mientras que la otra vacuna tenía una eficacia superior a 70\%. En diciembre 2020 la FDA autorizó dos vacunas para COVID-19 a saber, la Pfizer-BioNTech COVID-19 y la vacuna Moderna COVID-19. ${ }^{2}$ Para febrero 2021 la FDA aprobó la de Johnson and Johnson COVID-19. ${ }^{3}$ Dado que la vacuna contra el SARS-COV-2 es una nueva de $\mathrm{ARNm}$, el desarrollo tan rápido tendrá una mayor resistencia a recibirla. Una vacuna de ARNm se basa en introducir una secuencia de ARNm que codifica un antígeno específico de una enfermedad dentro del cuerpo que activa el sistema inmunológico. Cuando el sistema inmune está activado una vez expuesto a esa enfermedad, la respuesta inmunitaria sería más rápida y eficaz. Las razones del rechazo podrían estar relacionadas con el control de calidad, los efectos secundarios, el miedo a contraer COVID-19 y la eficacia. Muchas personas esperan que otros se vacunen primero. ${ }^{4-8}$ La cobertura que brinda protección a toda la comunidad fue desafiada a nivel mundial por la vacilación de las vacunas. ${ }^{9}$ El concepto de vacilación en vacunarse se refiere al rechazo o al retraso a tomarla a pesar de la existencia de vacuna. ${ }^{10}$

Es desafortunado que la vacilación en vacunarse se documentó incluso entre trabajadores de la salud en todo el mundo. ${ }^{10,11}$ El objetivo de esta investigación es exponer el avance de los esquemas completos aplicados contra SARSCOV-2 por grupo de edad en las entidades territoriales de Colombia, en el periodo comprendido entre el 17 de febrero al 21 de agosto 2021 .

\section{METODOLOGÍA}

Estudio de tipo transversal analítico diseñado específica para recoger información sobre las vacunas aplicadas en Colombia. La fuente de información fue el plan nacional de vacunación contra el COVID-19 de la página web del Ministerio de Salud y Protección Social ${ }^{12}$, en el periodo comprendido entre el 17 de febrero 2021 al 22 de agosto 2021. Además, se manejaron medios matemáticos y estadísticos cotidianos para valorar los resultados de modo evidente. ${ }^{13-15}$

\section{RESULTA D OS}

En la figura 1 podemos apreciar los valores de los esquemas completos por grupo de edad en los diferentes entes territoriales de Colombia al 21 de agosto 2021. En ella se puede inferir que las que cuentan con mayor número de esquemas completos por grupo de edad fueron: Bogotá, Antioquia, Valle del Cauca, Cundinamarca y Santander, y las de menor: Vaupés, Vichada, Guainía, Guaviare y Amazonas. 


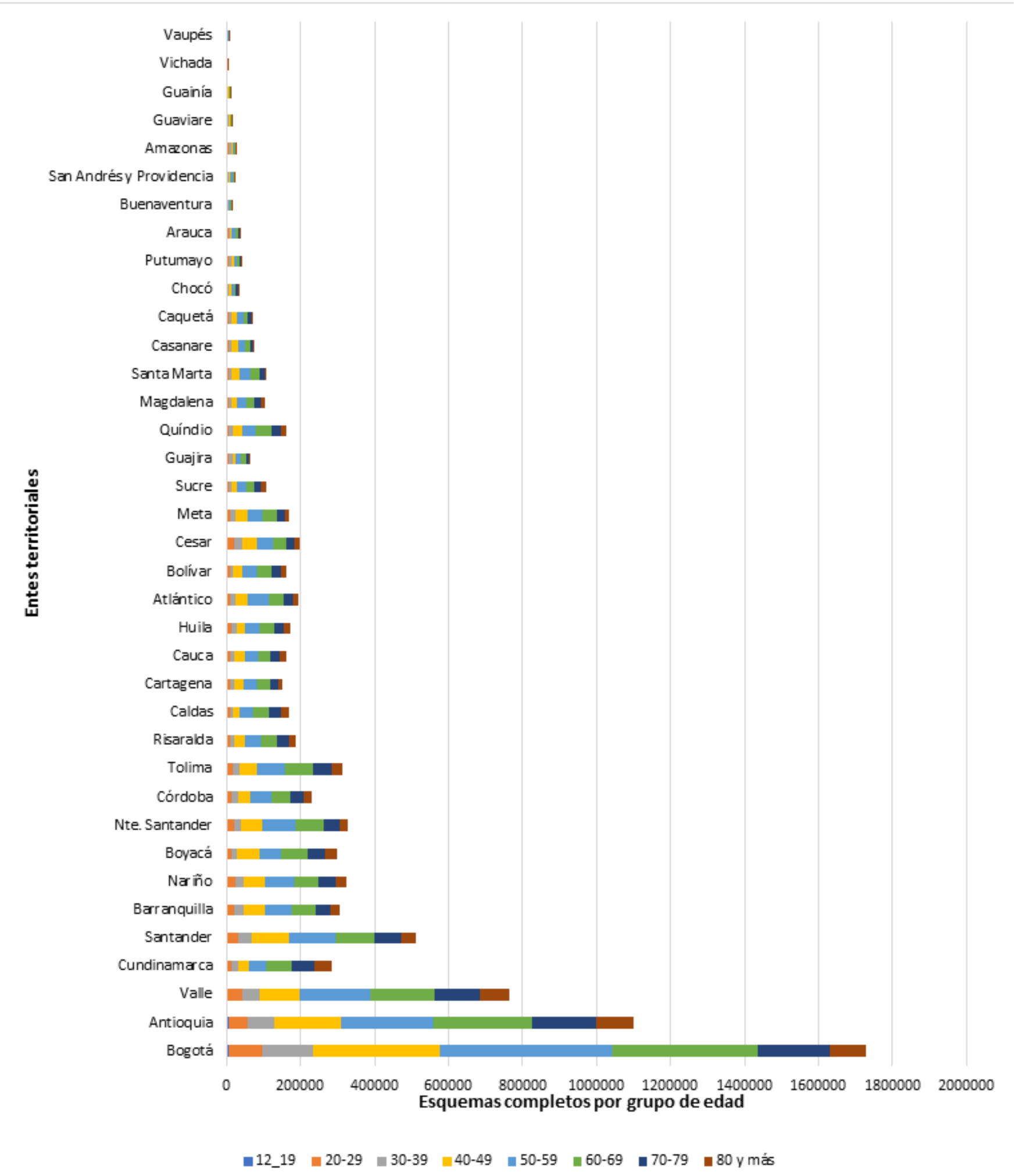

Fuente: el autor.

Figura 1. Esquemas completos por grupo de edad en entidades territoriales. 
La figura 2 muestra la totalidad de esquemas completos por grupo de edad para la vacunación contra SARS-CoV-2 en las entidades territoriales de Colombia al 21 de agosto 2021. Se puede concluir que el mayor número de esquemas completos por grupo de edad son: Bogotá (1'727.406), Antioquía (1'100.276), Valle del Cauca (763.135), Santander (510.054) y Norte de Santander (328.807) y el menor: Vichada (4.226), Vaupés (5.408), Guainía (8.664), Guaviare
(14.656) y Buenaventura (15.742).

En la figura 3 se observan los valores de la totalidad de esquemas completos por grupo de edad para la vacunación contra SARS-COV-2. El mayor número por grupo de edad es: 50 a 59 años (2'089.980), 60 a 69 años (1'937.880), y 40 a 49 años (1'480.711) y el menor número: 12 a 19 años (55.113), 20 a 29 años (476.866) y 30 a 39 años (624.909).

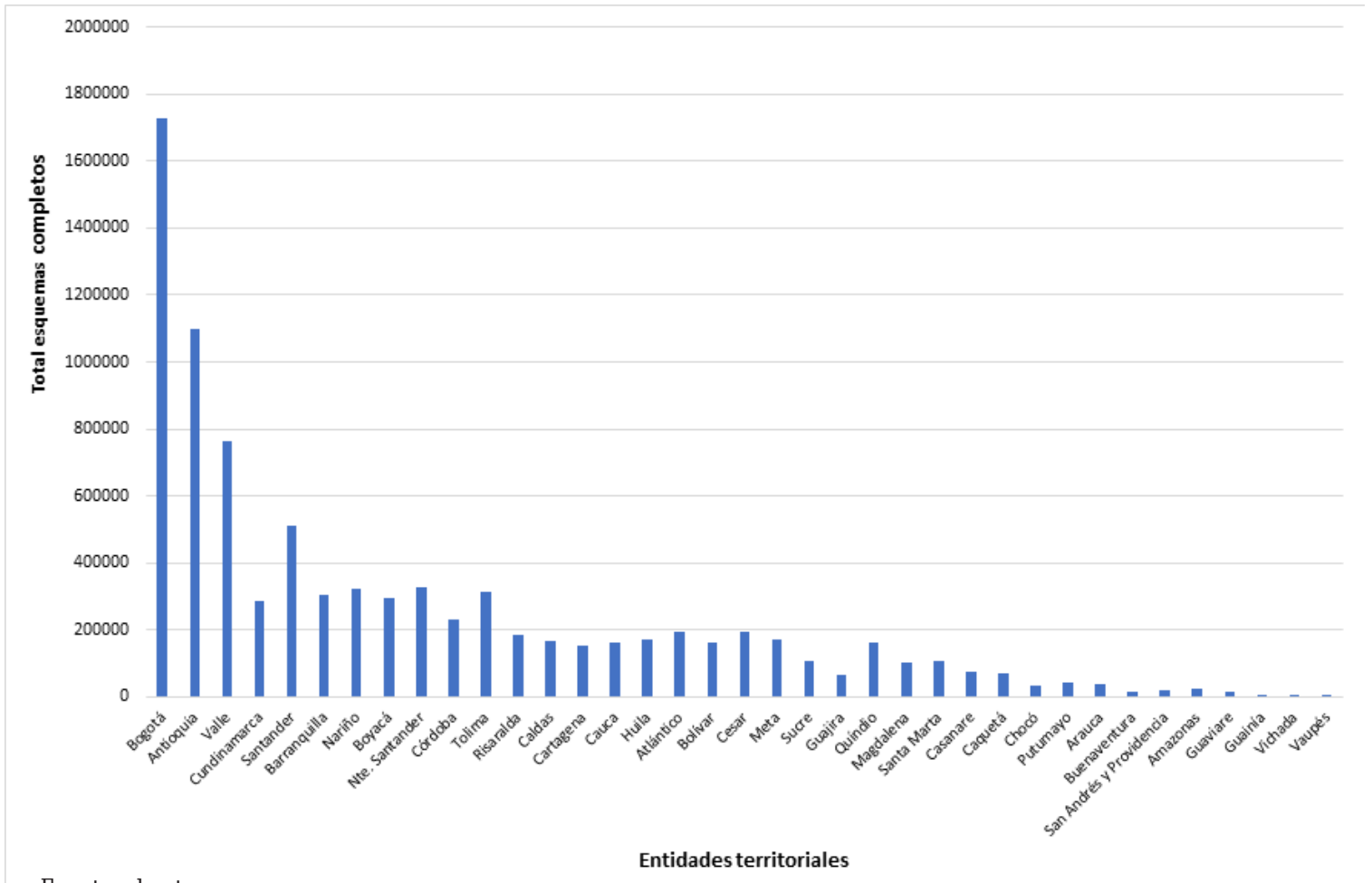

Fuente: el autor.

Figura 2. Total de esquemas completos por entidad territorial.

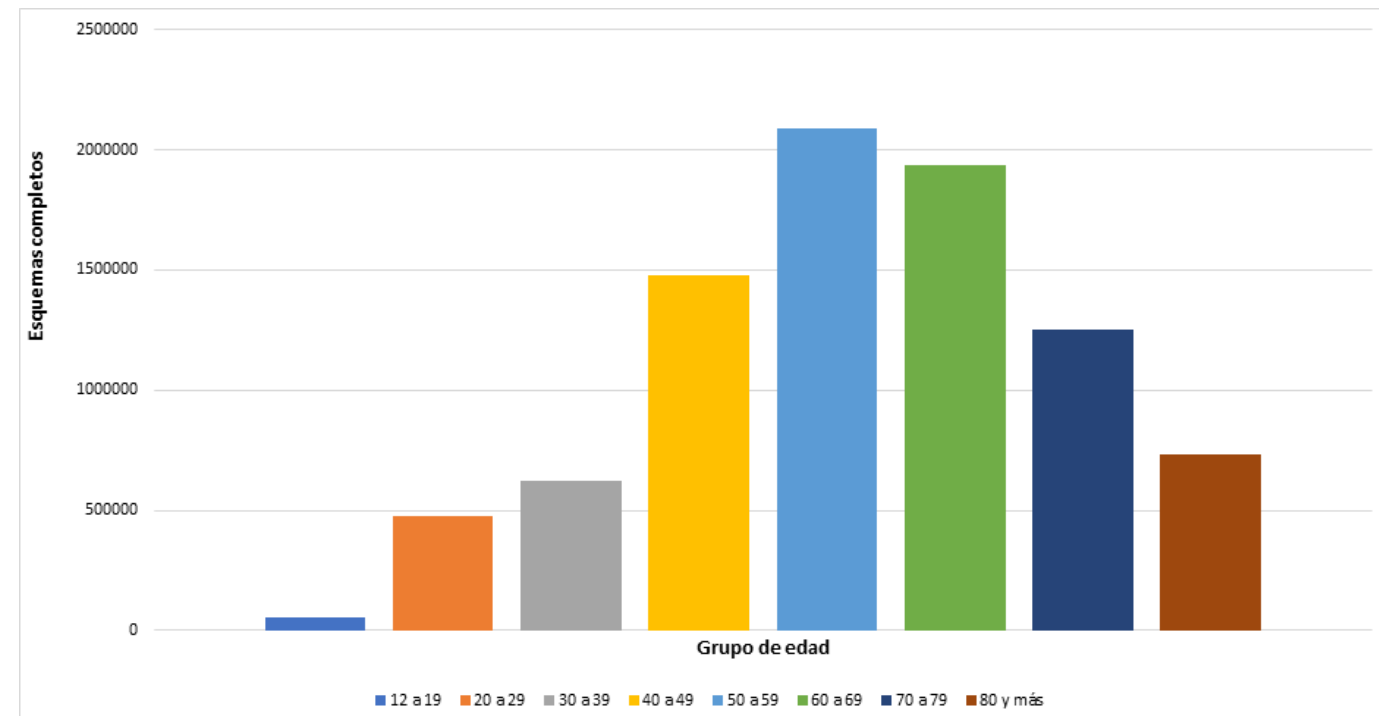

Fuente: el autor.

Figura 3. Esquemas completos por grupo de edad. 


\section{CONCLUSIONES}

Se estableció que a 21 de agosto 2021 las entidades territoriales de Colombia con el mayor número de esquemas completos por grupo de edad fueron: Bogotá (1'727.406), Antioquía (1'100.276), Valle del Cauca (763.135), Santander (510.054) y Norte de Santander (328.807). Además, se concluyó que el mayor número de esquemas completos por grupo de edad fueron: 50 a 59 años (2'089.980), 60 a 69 años (1'937.880) y 40 a 49 años (1'480.711), y el menor: 12 a 19 años (55.113), 20 a 29 años (476.866) y 30 a 39 años (624.909). En la actualidad la vacunación es la maniobra más importante para poner fin a la pandemia. Sin embargo, el arribo de múltiples variantes del SARS-COV-2 con susceptibilidad reducida a la inmunidad inducida por la vacuna y la enfermedad conmina el progreso. A pesar de estas amenazas continuas, la eficacia de las vacunas contra el SARS-COV-2 ofrece una verdadera esperanza para el $2021 .^{16}$

\section{DECLARACIÓN CONFLICTO} DE INTERESES

El autor declara no tener ningún conflicto de intereses.

\section{REFEREN CI AS}

1. Callaway E. The race for coronavirus vaccines: a graphical guide. Naturaleza. Nature. 2020;580(7805):576-577. doi: 10.1038/d41586-020-01221-y

2. Declaración de la FDA sobre el seguimiento de los programas de dosificación autorizados para las vacunas COVID-19 [Interne]. 2021 [citado 26 enero de 2021]. Disponible en: https://www.fda. gov/news-events/press-announcements/declaracion-de-la-fdasobre-el-seguimiento-de-los-programas-de-dosificacion-autorizados-para-las

3. Vi C Do T, Thota Kammili S, Reep M, Wisnieski L, Shyam Ganti S, Depa J. COVID-19 Vaccine Acceptance Among Rural Appalachian Healthcare Workers (Eastern Kentucky/West Virginia): A CrossSectional Study. Cureus. 2021;13(8):e16842. doi: 10.7759/cureus.16842

4. Dror AA, Eisenbach N, Taiber S, Morozov NG, et al. Vaccine hesitancy: the next challenge in the fight against COVID-19. Eur J Epidemiol. 2020;35(8):775-779. doi: 10.1007/s10654-020-00671-y

5. Wang J, Jing R, Lai X, Zhang H, Lyu Y, Knoll MD, Fang H. Acceptance of COVID-19 Vaccination during the COVID-19 Pandemic in China. Vaccines (Basel). 2020;8(3):482. doi: 10.3390/ vaccines 8030482
6. Neumann-Böhme S, Varghese NE, Sabat I, Pita Barros P, et al. Once we have it, will we use it? A European survey on willingness to be vaccinated against COVID-19. Eur J Health Econ. 2020;21(7):977982. doi: 10.1007/s10198-020-01208-6

7. Fisher KA, Bloomstone SJ, Walder J, Crawford S, Fouayzi H, Mazor KM. Attitudes Toward a Potential SARS-CoV-2 Vaccine : A Survey of U.S. Adults. Ann Intern Med. 2020;173(12):964-973. doi: 10.7326/M20-3569

8. Reiter PL, Pennell ML, Katz ML. Acceptability of a COVID-19 vaccine among adults in the United States: How many people would get vaccinated? Vaccine. 2020;38(42):6500-6507. doi: $10.1016 /$ j.vaccine.2020.08.043

9. Dror AA, Eisenbach N, Taiber S, Morozov NG, Mizrachi M, Zigron A, et al. Vaccine hesitancy: the next challenge in the fight against COVID-19. Eur J Epidemiol. 2020;35(8):775-779. doi: 10.1007/ s10654-020-00671-y

10. The European Centre for Disease Prevention and Control (ECDC). Vaccine hesitancy [Internet]. European Centre for Disease Prevention and Control; 2017 [cited 2021 Jun 27]. Available from: https://www.ecdc.europa.eu/en/immunisation-vaccines/vaccinehesitancy

11. Karafillakis E, Dinca I, Apfel F, Cecconi S, Wrz A, Takacs J, et al. Vaccine hesitancy among healthcare workers in Europe: A qualitative study. Vaccine. 2016;34(41):5013-20. doi: 10.1016/j. vaccine.2016.08.029

12. Ministerio de Salud y Protección Social de Colombia. Plan de vacunación nacional contra COVID-19 [Internet]. 2021 [citado 27 enero 2021]. Disponible en: https://www.minsalud.gov.co/salud/ publica/Vacunacion/Paginas/Vacunacion-covid-19.aspx

13. Díaz Pinzón JE. Estimación de la prevalencia del COVID-19 en Colombia. Repert Med Cir. 2020;29(Núm. Supl.1):99-102. https:// doi.org/10.31260/RepertMedCir.01217372.1115

14. Díaz Pinzón JE. Análisis de los resultados del contagio del COVID-19 respecto a su distribución geográfica en Colombia. Repert Med Cir. 2020;29(Núm. Supl.1):60-64. https://doi.org/10.31260/ RepertMedCir.01217372.1082

15. Díaz Pinzón JE. Dinámica y relación del contagio del COVID-19 después de iniciado el plan de vacunación contra el SARS-COV-2 en Colombia. Repert Med Cir. 2021;30(Núm. Supl.1):41-45. https://doi.org/10.31260/RepertMedCir.01217372.1227

16. Creech CB, Walker SC, Samuels RJ. SARS-CoV-2 Vaccines. JAMA. 2021;325(13):1318-1320. doi: 10.1001/jama.2021.3199 